\title{
Comparison of E-cadherin and CD44 Markers Expression in Oral Lichen Planus, Oral Leukoplakia and Oral Squamous Cell Carcinoma
}

\author{
Khadijeh Abdal $^{1}$, Samira Mostafazadeh ${ }^{2}$, Nastaran Ghorbani ${ }^{3}$
}

\begin{abstract}
Background: Lichen planus and leukoplakia may change dysplastically over time and are considered as premalignant lesions. CD44 and E-cadherin markers appear to have high potential in the premalignant evaluation of oral leukoplakia and lichen planus lesions. Therefore, the aim of this research was to compare the expression of CD44 and E-cadherin markers in oral leukoplakia and lichen planus and oral squamous cell carcinoma. Materials and methods: This analytical-descriptive research was conducted on 60 blocks of lichen planus, leukoplakia, and squamous cell carcinoma. The blocks were stained by CD44 and E-cadherin antibodies. The data obtained from this research were evaluated by SPSS 22.

Results: Only $30 \%$ of squamous cell carcinoma (SCC) samples expressed CD44 marker, while $40 \%$ and $50 \%$ of leukoplakia and lichen planus samples expressed CD44 marker. The expression of E- cadherin marker in SCC samples was $40 \%$ in the range of staining, while it was $50 \%$ and $60 \%$, respectively in leukoplakia and lichen planus. The intensity of staining was estimated to be equally severe in leukoplakia and lichen planus samples, and there was not a significant difference between the staining intensity of CD44 and E-cadherin $(p<0.16)$. While in SCC, 70\% of the cases showed mild to moderate expression intensity, while was statistically significant compared to lichen planus and leukoplakia ( $p<0.004)$. Conclusion: It seems that the severity of CD44 and E-cadherin incidence can indicate the changes in dysplasia and pre-malignancy of oral lichen planus and leukoplakia associated with oral carcinomas.

Keywords: CD 44, E-cadherin, Leukoplakia, Lichen planus, SCC.

CODS Journal of Dentistry (2021): 10.5005/jp-journals-10063-0080
\end{abstract}

\section{INTRODUCTION}

Oral squamous cell carcinoma (OSCC) is the most common malignancy of the oral cavity that originates, in the dysplastic superficial epithelium. Dysplasia is a change that begins in the basal and Para basal parts of the epithelium. ${ }^{1}$ The presence of severe epithelial dysplasia indicates a significant risk of malignancy. ${ }^{2}$

Leukoplakia is a white spot or plaque that is clinically and pathologically unlike any other lesion. ${ }^{3}$ Approximately $5 \%$ of leukoplakia samples are malignant at the time of the first biopsy and the remaining $5 \%$ undergo subsequent malignancy. ${ }^{4}$ About $10-15 \%$ of dysplasia diagnosed as leukoplakia eventually develops squamous cell carcinoma, especially in the floor of the mouth. ${ }^{5}$

Lichen planus is a chronic inflammatory disease of the skinmucosa that often involves the oral cavity and occurs mainly in people aged $30-70$ years and in women. ${ }^{6}$ Its prevalence in different populations has been reported between 0.5 and $2.3 \%{ }^{7}$ An important issue with Lichen planus is the possibility of malignant transformations. ${ }^{8}$ This issue has been debated for many years. Although extensive research has been done in this area and a specific lesion has been identified as a separate premalignant lesion, it is still questionable whether the lesion is benign or prone to malignancy. ${ }^{9}$ Many oral squamous cell carcinomas have been reported to begin with a premalignant lesion, especially leukoplakia. ${ }^{10}$ Malignant changes in OLP have been reported up to $10 \%$. The association between an increased risk of malignancy and a specific type of OLP lesion is unclear. ${ }^{11}$ However, some studies have found an increased risk of squamous cell carcinoma in atrophic and erosive types and others in plaque-like form. According, the oral lichen planus has been defined by the World Health Organization as
${ }^{1}$ Department of Oral and Maxillofacial Pathology, Faculty of Dentistry, Ilam University of Medical Sciences, Ilam, Iran

${ }^{2}$ Department of Oral and Maxillofacial Pathology, Faculty of Dentistry, Urmia University of Medical Sciences, Urmia, Iran

${ }^{3}$ Department of Orthodontics, Faculty of Dentistry, Ilam University of Medical Sciences, Ilam, Iran

Corresponding Author: Samira Mostafazadeh, Department of Oral and Maxillofacial Pathology, Faculty of Dentistry, Urmia University of Medical Sciences, Urmia, Iran, Phone: +91 44153104, e-mail: dr.faribaabdal@yahoo.com

How to cite this article: Abdal K, Mostafazadeh S, Ghorbani N. Comparison of E-cadherin and CD44 Markers Expression in Oral Lichen Planus, Oral Leukoplakia and Oral Squamous Cell Carcinoma. CODS J Dent 2021;13(1):6-10.

Source of support: Nil

Conflict of interest: None

a premalignant condition. ${ }^{12}$ Oral leukoplakia, OLP, and OSCC are the most common oral diseases with specific clinical and histological features and in most cases are associated with chronic inflammation in adjacent connective tissue. ${ }^{13}$ Decreased or increased expression of some molecular-protein markers in suspected malignant lesions has been somewhat effective in the early detection of oral cancer. Among many protein factors, two are clinically important including CD44 and E-cadherin. ${ }^{14}$ CD44 is a membrane glycoprotein with various isoforms of $\mathrm{V} 10$ to $\mathrm{V} 10$ that results from different mRNA expression..$^{15}$ The decrease of CD44 is due to ectodomain fracture, which occurs in a large number of malignant tumors. CD44 fractures

(c) The Author(s). 2021 Open Access This article is distributed under the terms of the Creative Commons Attribution 4.0 International License (https://creativecommons. org/licenses/by-nc/4.0/), which permits unrestricted use, distribution, and non-commercial reproduction in any medium, provided you give appropriate credit to the original author(s) and the source, provide a link to the Creative Commons license, and indicate if changes were made. The Creative Commons Public Domain Dedication waiver (http://creativecommons.org/publicdomain/zero/1.0/) applies to the data made available in this article, unless otherwise stated. 
are said to separate tumor cells from the extracellular matrix and lead to tumor cells migration, tumor spread, and metastasis. ${ }^{16} \mathrm{E}$ cadherin is calcium-dependent membrane glycoprotein that is responsible for cell-to-cell binding in the epithelium. E-cadherins make connections in epithelial tissues thus keeping the epithelial cells together. ${ }^{17}$ Many epithelial neoplasms, including oral squamous cell carcinoma (OSCC), show the changes in the expression of $\mathrm{E}$-cadherin protein. The possibility of this change in extent causes a defect in the ability of the cells to attach to each other and facilitates separation from the primary tumor and attachment to the substrate components and progression to the surrounding tissues. ${ }^{8,11}$ Given the role of $\mathrm{CD} 44$ and $\mathrm{E}$-cadherin in the progression of cancerous and precancerous lesions, it seems that these two markers have a high potential in assessing the precancerous lesions of oral leukoplakia and lichen planus. ${ }^{3,10}$ Therefore, the aim of this research was to evaluate and compare the expression of CD44 and E-cadherin markers in oral leukoplakia and lichen planus lesions as well as their progression to OSCC.

\section{Materials and Methods}

This research is retrospective and the statistical population includes blocks related to patients with oral lichen planus, leukoplakia and OSCC in the archive of the pathology department of Ilam university of Medical sciences.

According to the study of Asareh and according to the formula for determining the sample size of the prevalence studies that is described below, the sample size of 60 cases was estimated in each group. ${ }^{4}$ By placing these numbers in the formula and adding $10 \%$ due to the drop, the sample size was 60 people.

To analyze the data obtained from this study, descriptive statistics including frequency and percentage were applied using chi-square test for qualitative data. The normality of the data was measured by Kolmograph-Smirnov test. If the data were normal, ANOVA test would use, and if they were not normal, Kruskal-Wallis test would use to compare the three groups. SPSS 22 software was used, and a significance level of 0.05 was considered.

\section{Method}

Initially, existing slides stained by H\&E were examined by a pathologist and the slides were separated by diagnosis of oral leukoplakia, oral lichen planus, and OSCC. Then the blocks related to the slides were collected and evaluated in terms of the amount of tissue required for immune histochemical $(\mathrm{IHC})$ staining.

The blocks were stained by CD44 and E-cadherin antibody kits, then 5-micron incisions were made and new slides that were stained by IHC staining were examined by a pathologist. In this research, normal epithelial tissue was used as a positive control and lack of primary antibody was used as a negative control.

The following codes are defined to evaluate the intensity of staining: ${ }^{4}$

Code 0: unstained

Code 1 : less than $25 \%$ staining
Code 2: $25-50 \%$ staining

Code $3: 50-75 \%$ staining

Code 4 : over $75 \%$ staining

\section{The thickness of the stained epithelium is as follows ${ }^{4}$ :}

Code 0 : lack of staining

Code 1: staining up to one-third of the epithelium

Code 2: staining up to two-thirds of the epithelium

Code 3: staining of the entire thickness of the epithelium

\section{Ethical Statement}

The protocol of this study was approved by the Ethics Committee of Ilam University of Medical Sciences, Ilam, Iran (Ethical code: IR.MEDILAM.REC.1399.254). Written informed consent was obtained from all participants included in the study.

\section{Results}

This research was conducted on 60 blocks including 20 SCC, 20 lichen planus, and 20 leukoplakia in the field of expression of CD44 and E-cadherin markers. There were 10 men and 10 women in each group. Equally, each marker included 5 men and 5 women.

Intensity and extent of samples' staining with CD44 and E-cadherin markers are presented in Tables 1 to 4 separately for all three groups.

Examination of the staining range of SCC, lichen planus, and leukoplakia samples in the field of CD44 marker expression showed that $30 \%$ of SCC samples, $40 \%$ of leukoplakia samples and $50 \%$ of lichen planus samples had a staining range of $50-75 \%$ (Fig. 1). According to the results of Fisher's exact test, there was not a statistically significant difference between the three groups of SCC, lichen planus, and leukoplakia in terms of the percentage of stained cells (the extent of staining) ( $p=0.16)$.

In terms of staining of epithelial thickness in the samples studied by CD44 marker, the whole thickness was stained in SCC sample in $20 \%$ of cases. In leukoplakia and lichen planus samples in $60 \%$ and $80 \%$ of cases, two-thirds of the epithelial thickness was stained, respectively. According to the results of fisher's exact test, there was a statistically significant difference between the three groups (SCC, lichen planus, and leukoplakia) in terms of thickness of the stained epithelium ( $p=0.004)$.

Staining intensity CD44 in SCC samples was at weak level in 70\% of the cases, and it was at severe level in $50 \%$ of leukoplakia and lichen planus samples. Statistical analysis of data using fisher's exact test did not show a statistically significant difference between the three group (SCC, leukoplakia, and lichen planus) in terms of staining intensity $(p=0.07)$.

Regarding the extent of staining of SCC, leukoplakia, and lichen planus samples to express E-cadherin marker, the findings showed that the extent of staining in SCC samples was over $75 \%$ in $10 \%$ of samples, and it was $50 \%$ and $60 \%$ in leukoplakia and lichen planus, respectively (Fig. 2). The results of Fisher's exact test to examine the percentage of stained cells (the extent of staining) in the three

Table 1: Frequency distribution of staining intensity in the studied groups (CD44 marker)

\begin{tabular}{lccccc}
\hline Rank & Unstained & Mild & Moderate & Severe & Test result (P) \\
\hline SCC & 0 & $4(40)$ & $4(40)$ & $2(20)$ & 0.24 \\
Leukoplakia & 0 & $4(40)$ & $2(20)$ & $4(40)$ & $5(50)$ \\
Lichen planus & 0 & 0 & $5(50)$ & & \\
\hline
\end{tabular}


Table 2: Thickness of the stained epithelium in the studied groups (CD44 marker)

\begin{tabular}{llllll}
\hline Thickness & 0 & 1 & 2 & 3 & Test result (P) \\
\hline OSCC & 0 & 0 & $8(80)$ & $2(20)$ & 0.004 \\
Leukoplakia & 0 & 0 & $4(40)$ & $6(60)$ & $8(80)$ \\
Lichen planus & 0 & 0 & $2(20)$ & & \\
\hline
\end{tabular}

Table 3: Frequency distribution of staining intensity in the studied groups (E-cadherin marker)

\begin{tabular}{lccccc}
\hline Rank & Unstained & Mild & Moderate & Severe & Test result (P) \\
\hline SCC & 0 & $6(60)$ & $3(30)$ & $1(10)$ & 0.07 \\
Leukoplakia & 0 & $5(50)$ & $1(10)$ & $4(40)$ & $4(40)$ \\
Lichen planus & 0 & $2(20)$ & $4(40)$ & \\
\hline
\end{tabular}

Table 4: Thickness of the stained epithelium in the studied groups (E-cadherin marker)

\begin{tabular}{llllll}
\hline Thickness & 0 & 1 & 2 & 3 & Test result (P) \\
\hline OSCC & 0 & $6(60)$ & $2(20)$ & $2(20)$ & 0.08 \\
Leukoplakia & 0 & $2(20)$ & $2(20)$ & $6(60)$ & $5(50)$ \\
Lichen planus & 0 & $1(10)$ & $4(40)$ & & \\
\hline
\end{tabular}

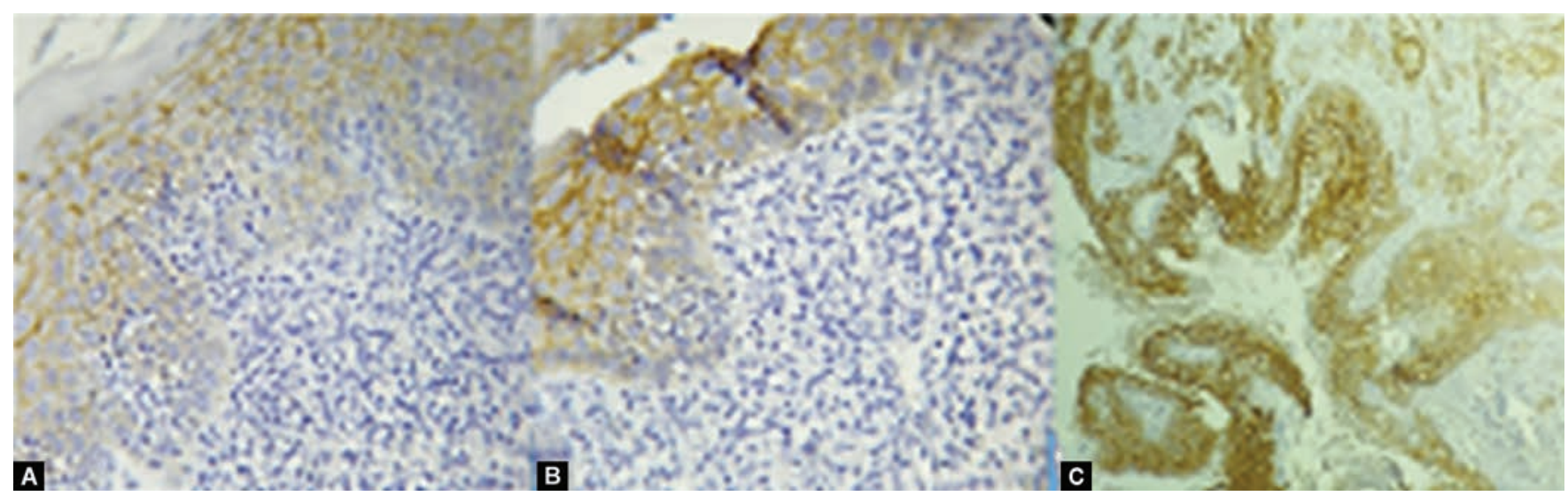

Figs. $1 \mathrm{~A}$ to C: CD44 immunostaining in studied groups. CD44 expression in: (A) Oral lichen planus, $\times 10$ magnification; (B) Leukoplakia $\times 10$ magnification; (C) Squamous cell carcinoma $\times 10$ Magnification

groups (SCC, leukoplakia and lichen planus) showed a statistically significant difference $(p=0.006)$.

The entire thickness of the epithelium was stained by E-cadherin marker in $20 \%$ of SCC samples. In leukoplakia samples $60 \%$ and in lichen planus samples $50 \%$ of cases showed the staining of the entire thickness of the epithelium. These findings showed a statistically significant difference $(p=0.008)$.

\section{Discussion}

In the study of Asareh et al., the staining intensity of CD44 in lichen planus and dysplastic epithelium was over $70 \% .{ }^{4}$ In addition, the results of Zargaran et al.'s research were acceptable regarding the expression of CD44 marker in lichen planus that are consistent with the results of present research. ${ }^{17}$

The results of Neppelberg et al.'s research showed that CD44 marker expression was acceptable in areas with oral lichen planus in epithelial tissues as well as areas with compact tissue, which is consistent with the results of our research. ${ }^{18}$ The findings of Chaiyarit et al.'s research showed that CD44 is a desirable indicator for the diagnosis of oral lichen planus from other epithelial dysplastic lesions that is consistent with the results of our research. ${ }^{19}$
Regarding the expression of E-cadherin marker, the results of Yong et al.'s research showed that this factor is expressed $50 \%$ less in people with lichen planus than healthy people, so this marker is a good indicator for diagnosing lichen planus, which is consistent with the findings of our research. ${ }^{20}$

In the study of Neppelberg et al., which examined the expression of E-cadherin marker in people with oral lichen planus, the expression of E-cadherin marker in areas with lichen planus in epithelial tissues was satisfactory and acceptable. ${ }^{18}$ However, in sub-epithelial areas and areas with dense tissue, there was a focal decrease in E-cadherin expression, which contradicts the results of the present research.

Regarding the extent of staining of epithelial thickness in the samples studied by CD44 marker, in Asareh et al.'s research on staining of epithelial thickness in $100 \%$ of cases, two thirds of the thickness of the epithelium were stained, which was consistent with the findings of our research. ${ }^{4}$

In the research of Bahar et al., about $100 \%$ of SCC samples showed a decrease of expression of CD44 marker. ${ }^{21}$ The results of Simionescu et al.'s research showed that the lower the distinction and the higher the grade of SCC, the lower the intensity of CD44 marker expression that is consistent with the results of present research. ${ }^{22}$ 


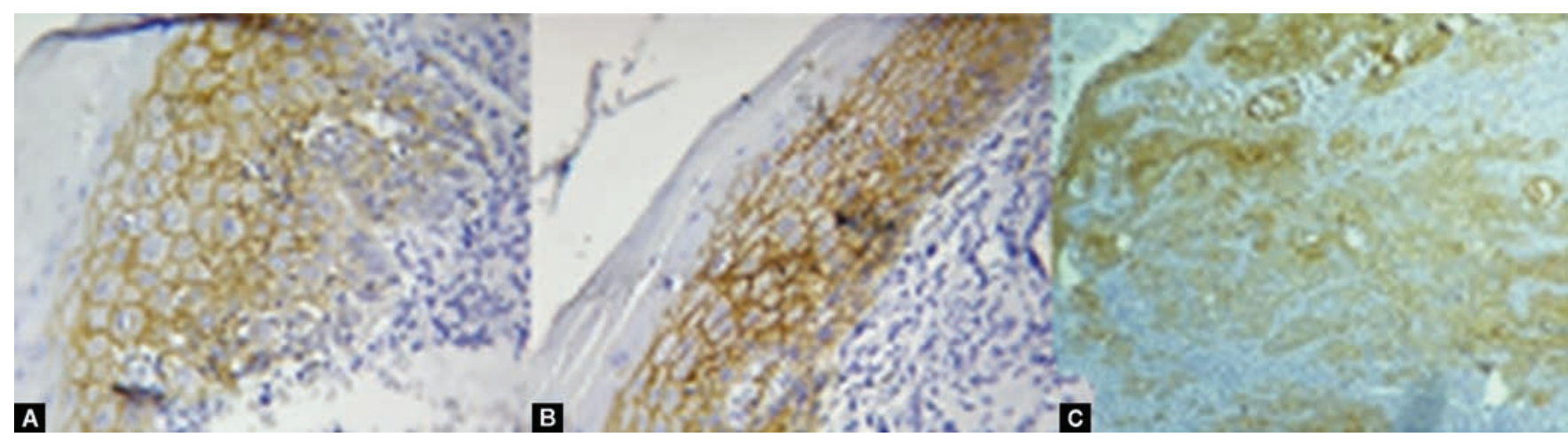

Figs. 2 A to C: E-cadherin immunostaining in studied groups. E-cadherin expression in: (A) Oral lichen planus, $\times 10$ magnification; (B) Leukoplakia $\times 10$ magnification; (C) Squamous cell carcinoma $\times 10$ Magnification

The results of Abdulmajeed and Mannelli's research showed that the expression of CD44 and E-cadherin markers increases in dysplastic and SCC lesions, which contradicts the findings of present research. ${ }^{23,24}$ While it is expected that in cases where epithelial tissue undergoes dysplastic changes, intercellular connections are reduced and in cases where carcinoma such as SCC invades the underlying tissues, interconnection between cells is completely lost, thus factors that are effective in intercellular connections such as CD44 and E-cadherin show less intensity of expression. ${ }^{4,7}$ In the present research, the expression intensity of both of these factors in SCC was lower than leukoplakia and lichen planus, which shows the same thing. There was not a significant difference between the staining intensity of markers in lichen planus and leukoplakia, but the expression of these markers was lower in SCC. Therefore, it can be said that these two protein factors are somewhat effective in dysplastic changes and malignant potential of lichen planus and leukoplakia, so it is suggested that more research be done in this field.

\section{ConcLusion}

Based on the findings of this research, there was a significant difference between the extent of expression of CD44 and E-cadherin in leukoplakia and lichen planus compared to SCC and it seems that the extent of expression of these two proteins can indicate the changes in dysplasia and precancerous lesions of leukoplakia and lichen planus in oral carcinomas.

\section{Acknowledgment}

This article is an exception from the dissertation of the General Doctor of Dentistry. The authors would like to thank llam University of Medical Sciences for supporting us in this research.

\section{References}

1. MoradzadehKhiavi M, Anvari E, Hamishehkar H, et al. Assessment of the blood parameters, cardiac and liver enzymes in oral squamous cell carcinoma following treated with injectable doxorubicin-loaded nano-particles. Asian Pac J Cancer Prev 2019;20(7):1973-1977. DOI: 10.31557/apjcp.2019.20.7.1973

2. Seif S, Jafari-ashkavandi Z, Mardani M, et al. Evaluation of serum vitamin d level in oral lichen planuspatients. J Mash Dent Sch 2018;42(1):58-49.

3. Kalbasi GN, Afshar MN, Dehghan DH, et al. Relationship between E-cadherin protein expression and oral squamous cell carcinoma. Grading 2019;15(3):273-282.
4. Asareh F, Shirvani S. Immunohistochemical expression of CD44 in erosive lichen planus, epithelial dysplasia, and oral squamous cell carcinoma. J Mash Dent Sch 2018;42(1):66-59.

5. Khiavi MM, Abdal K, Abbasi MM, et al. Comparison of injectable doxorubicin \& its nanodrug complex chemotherapy for the treatment of 4-nitroquinoline-1-oxide induced oralsquamous cell carcinoma in rats. Indian J Med Res 2017;145:112-117. DOI: 10.4103/ijmr. IJMR_542_14

6. Fakhry C, Lacchetti C, Rooper LM, et al. Papillomavirus testing in head and neck carcinomas: ASCO clinical practice guideline endorsement of the college of American pathologists guideline. J Clin Oncol 2018;36(31):3152-3161. DOI: 10.1200/JCO.18.00684

7. Lewis Jr. JS, Beadle B, Bishop JA, et al. Human papillomavirus testing in head and neck carcinomas: guideline from the college of American pathologists. Arch Pathol Lab Med 2018;142(5):559-597. DOI: 10.5858/arpa.2017-0286-CP

8. Sridevi U, Jain A, Nagalaxmi V, et al. Expression of E-cadherin in normal oral mucosa, in oral precancerous lesions and in oral carcinomas. Eur J Dent 2015;9(3):364-372. DOI: 10.4103/1305-7456.163238

9. Mostafazadeh S, Emamverdizadeh P, Abdal K, et al. A comparative study of the frequency of myofibroblasts and macrophages between the oral and cutaneous squamous cell carcinoma. J Dent Res Dent Clin Dent Prospects 2019;13(4): 253-257. DOI: 10.15171/joddd.2019.039

10. Mello FW, Miguel AFP, Dutra KL, et al. Prevalence of oral potentially malignant disorders: a systematic review and meta-analysis. J Oral Pathol 2018;47(7):633-640. DOI: 10.1111/jop.12726

11. Van der Waal I. Oral leukoplakia, the ongoing discussion on definition and terminology. Med Oral Patol Oral Cir Bucal 2015;20(6):e685. DOI: 10.4317/medoral.21007

12. Arduino PG, Bagan J, El-Naggar AK, et al. Urban legends series: oral leukoplakia. Oral Dis 2013;19(7):642-459. DOI: 10.1111/odi.12065

13. Anderson A, Ishak N. Marked variation in malignant transformation rates of oral leukoplakia. Evid Based Dent 2015;16(4):102-103. DOI: 10.1038/sj.ebd.6401128

14. Van der Waal I. Oral potentially malignant disorders: is malignant transformation predictable and preventable? Med Oral Patol Oral Cir Bucal 2014;19(4):e386. DOI: 10.4317/medoral.20205

15. Speight PM, Khurram SA, Kujan O. Oral potentially malignant disorders: risk of progression to malignancy. Oral Surg Oral Med Oral Pathol Oral Radiol 2018;125(6):612-627. DOI: 10.1016/j. oooo.2017.12.011

16. Fonseca-Silva T, Diniz MG, de Sousa SF, et al. Association between histopathological features of dysplasia in oral leukoplakia and loss of heterozygosity. Histopathology 2016;68(3):456-460. DOI: $10.13039 / 501100003593$

17. Zargaran M, Baghaei F, Moghimbeigi A. Comparative study of $\beta$-catenin and CD44 immunoexpression in oral lichen planus and squamous cell carcinoma. Int J Dermatol 2018;57(7):794-798. DOI: $10.1111 / \mathrm{ijd}$.14007

18. Neppelberg E, Loro LL, Oijordsbakken G, et al. Altered CD40 and $\mathrm{E}$-cadherin expression-putative role in oral lichen planus. J Oral 
Pathol Med 2007;36(3):153-160. DOI: 10.1111/j.1600-0714.2007. 00511.x

19. Chaiyarit $P$, Thongprasom K, Satayut $S$, et al. Alteration of the exoression of CD44 isoform in oral epithelial and saliva from patient with oral lichen planus. J Clin Immunol 2008;28(1):26-34. DOI: 10.1007/s10875-007-9128-5

20. Du Y, Li H. Expression of E-cadherin in oral lichen planus. Exp Ther Med 2015;10(4):1544-1548. DOI: 10.3892/etm.2015.2654

21. Bahar R, Kunishi M, Kayada Y, et al. CD44 variant 6 (CD44v6) expression as a progression marker in benign, premalignant and malignant oral epithelial tissues. Int J Oral Maxillofac Surg 1997;26(6):443-446. DOI: 10.1016/s0901-5027(97)80010-0
22. Simionescu $C$, Mărgăritescu $C L$, Surpăţeanu $M$, et al. The study of $\mathrm{E}$-cadherine and CD44 immunoexpression in oral squamous cell carcinoma. Rom J Morphol Embryol 2008;49(2):189-193.

23. Dalley AJ, Abdul Majeed AA, Pitty LP, et al. LGR5 expression in oral epithelial dysplasia and oral squamous cell carcinoma. Oral Surg Oral Med Oral Pathol Oral Radiol 2015;119(4):436-440. DOI: 10.1016/j. oooo.2014.11.014

24. Mannelli G, Magnelli L, Deganello A, et al. Detection of putative stem cell markers, CD44/CD133, in primary and lymph node metastases in head and neck squamous cell carcinomas. A preliminary immunohistochemical and in vitro study. Clin Otolaryngol 2015;40(4):312-320. DOI: 10.1111/coa.12368 